\title{
STEINITZ CLASSES IN QUARTIC FIELDS
}

\author{
STEPHEN PIERCE ${ }^{1}$
}

Abstract. Let $K$ be normal quartic over the rationals. Let $l \equiv 3$ (4) be an odd prime. If the class number of $K$ is even, there is a normal extension $L$ of degree $l$ over $K$ such that the relative discriminant is principal, but $L$ has no relative integral base over $K$.

I. Introduction and results. Let $K$ be an algebraic number field, and $L$ a finite extension. The relative discriminant $D_{L / K}$ is an ideal of $K$. Let $d$ be the discriminant of a $K$-base of $L$ and $(d)$ the principal ideal generated by $d$. Then $D_{L / K}=B^{2}(d)$ for some fractional ideal $B$ of $K$. The ideal class to which $B$ belongs is written $C(L / K)$ and is called the Steinitz class of $L$ with respect to $K$.

Artin [1] showed that $L$ has a relative integral base over $K$ if and only if $C(L / K)$ is principal. Thus if the class number $h_{K}$ is odd, $L$ has a relative integral base if and only if $D_{L / K}$ is principal.

The story is different if $h_{K}$ is even; $C(L / K)$ may be in a class of order 2, i.e., $D_{L / K}$ can be principal without $L$ having an integral $K$-base.

Fröhlich [2] showed that every ideal class of $K$ is a Steinitz class for some quadratic extension. For a fixed odd prime $l$, Long [5] found which classes of $K$ can be Steinitz classes for some normal extension of degree $l$. We repeat his result. The classes are those of the form $C^{l-1 / 2}$, where $C$ is a class containing a prime divisor of $l$ or $C$ contains a prime which splits fully upon adjunction of the $l$ th roots of unity.

Let $K$ be an algebraic number field, and let $l$ be an odd prime. We say $K$ has property (*) with respect to $l$ if there is a normal extension $L$ of degree $l$ which has no relative integral base, but $D_{L / K}$ is principal.

No field $K$ with odd class number can have (*) with respect to any prime; $D_{L / K}$ is principal if and only if $L$ has a relative integral base. Thus, for the rest of the paper, we only deal with fields $K$ for which $h_{K}$ is even.

If $l \equiv 1(\bmod 4)$ and $h_{K}=2$, it is clear that $K$ does not have $(*)$ with respect to $l$. For the case $l \equiv 3$ (4) and $h_{K}$ even, the problem seems harder. We do not know of any such fields which do not have property (*) with respect to $l$.

Received by the editors April 16, 1973.

AMS (MOS) subject classifications (1970). Primary 12A30; Secondary 12A65.

Key words and phrases. Steinitz class, ideal class group.

${ }^{1}$ This work was partly supported by National Research Council of Canada Grant A-7862.

(c) American Mathematical Society 1974 
THEOREM 1. Let $K$ be quadratic over the rationals $Q$. Suppose $h_{K} i$ even and $l \equiv 3(4)$ is prime. Then $K$ has (*) with respect to $l$.

THEOREM 2. Let $K$ be normal quartic over $Q$. Suppose $h_{K}$ is even and $l \equiv 3$ (4) is prime. Then $K$ has $(*)$ with respect to $l$.

II Proofs. First, some preliminary remarks. The ideal classes of $K$ which are Steinitz classes for some normal extension of $K$ of degree $l$ form a group [5]. If $K$ does not have (*) then all primes which split fully upon adjunction of an $l$ th root of unity $\zeta$ are in classes of odd order. Thus the 2-part of the Hilbert class field of $K$ lies in $K(\zeta)$ and hence $h_{K} \equiv$ $2(\bmod 4)$. Also $K\left((-l)^{1 / 2}\right)$ is quadratic unramified over $K$ and $K$ is totally imaginary.

Theorem 1 is easy to complete. We have $K$ imaginary and $l \mid D_{K / Q}$. Now $K \neq Q\left((-l)^{1 / 2}\right)$, since $-l$ is not a square in $K$; thus $l$ is the square of a prime ideal in a class of order 2.

We divide Theorem 2 into two cases. First assume $K$ is cyclic over $Q$. Let $k$ be the unique subfield; $k$ is real. A prime fully ramified from $Q$ to $K$ is $\equiv 1$ (4) or is 2 . Any prime ramified in $k$ is fully ramified in $K$. Thus $l$ is ramified from $k$ to $K$.

Let $h_{0}$ be the narrow class number of $k$. Let $t$ be the number of primes (including infinite primes) ramifying from $k$ to $K$. By a formula of Hasse [3, p. 99], the number $h$ of ambiguous classes of $K$ over $k$ is

$$
h=h_{0} 2^{t+q^{*}-3}
$$

and the number $h^{\prime}$ of ambiguous classes of $K$ containing ambiguous ideals is

$$
h^{\prime}=h_{0} 2^{t+q-3}
$$

where $q^{*}, q$ are given by

$$
\begin{aligned}
2^{\alpha^{*}} & =\left(E_{k} \cap N_{K / k} K^{*}: E_{k}^{2}\right), \\
2^{\alpha} & =\left(E_{k} \cap N_{K / k} E_{K}: E_{k}^{2}\right) .
\end{aligned}
$$

In (3), (4), $E_{K}, E_{k}$ are the unit groups.

The ambiguous classes of $K$ are a group, and since $h_{K} \equiv 2$ (4), we also have $h \equiv 2(4)$. In the case of $K$ cyclic over $Q$, we have $t \geqq 4$ and hence $h_{0}$ is odd and $q^{*}=0$. Thus $k=Q\left(p^{1 / 2}\right)$ or $Q\left(2^{1 / 2}\right)$ and $p \equiv 1$ (4). Now $l$ is inert in $k$; otherwise $t \geqq 5$. Thus $D_{K / k}=\left(l p^{1 / 2}\right)$. It follows that $l$ is the square of a prime in the class of order 2 in $K$.

Next, let $K$ have Galois group $C_{2} \times C_{2}$. Let $k$ be the real subfield. Suppose $l$ ramifies from $Q$ to $k$. Then $2 \mid h_{0}$ and the fundamental unit $\varepsilon$ of $k$ has norm 1. Hasse's formula yields $t=3, q^{*}=0 ; t=2, q^{*}=1$; or $t=2$, 
$q^{*}=0$. Since $\varepsilon$ is totally positive, $\varepsilon$ is a norm at all primes except possibly one; hence $\varepsilon$ is a global norm and $q^{*} \geqq 1$. Our only alternative is $t=2$, $q^{*}=1, h_{0} \equiv 2$ (4). Then $K$ must be $k\left((-l)^{1 / 2}\right)$ which contradicts the fact that $-l$ is not a square in $K$.

Finally, suppose $l$ does not ramify in $k$. In (1), $t \geqq 3$ and our alternatives are $t=3, q^{*}=0 ; t=3, q^{*}=1 ; t=4, q^{*}=0$. If $q^{*}=1, t=3$, then $h_{0}$ is odd and $k=Q\left(p^{1 / 2}\right), p \equiv 1(4)$ a prime or $p=2$. In either case, $\varepsilon$ is not totally positive, so $q^{*} \neq 1$.

In the other cases, $q^{*}=q=0$. If $t=3, l$ is the only finite prime ramifying, so $l$ ramifies in the class of order 2 . If $t=4, h_{0}$ is odd and $k=Q\left(p^{1 / 2}\right)$ or $Q\left(2^{1 / 2}\right)$ as before. Then $k^{\prime}=Q\left((-l r)^{1 / 2}\right)$ is a subfield of $K$, where $r$ is a prime different from $p$. Thus $l, r$ are inert in $k$ and ramify from $k$ to $K$. Hence the prime in $K$ dividing $l$ must lie in the class of order 2 .

III. Additional remarks. It is clear that any normal extension $K$ of $Q$ with even class number must have property $(*)$ with respect to any odd prime $l$, simply because $l$ cannot have even ramification index in $K$.

If $l \equiv 3(4)$ and $K$ is an abelian field with even class number and not having property $(*)$, then the largest subfield of $K$ which has degree a power of 2 also does not have $(*)$.

\section{REFERENCES}

1. E. Artin, Collected works, Addison-Wesley, Reading, Mass., pp. 229-321.

2. A. Fröhlich, Discriminants of relative extensions and the existence of integral bases, Mathematika 7 (1960), 15-22. MR 27 \#1436.

3. H. Hasse, Bericht über neuere Untersuchungen und Probleme aus der Theorie der algebrasichen Zahlkörper, 1930.

4. H. B. Mann, On integral bases, Proc. Amer. Math. Soc. 9 (1958), 167-172. MR 20 \#26.

5. R. L. Long, Steinitz classes of cyclic extensions of prime degree, J. Reine Angew. Math. 250 (1971), 87-98. MR 44 \#6647.

Department of Mathematics, University of Toronto, Toronto M5S 1A1, CANADA 